\title{
Nasal nitric oxide levels in primary ciliary dyskinesia, cystic fibrosis and healthy children
}

\author{
Elif Güney ${ }^{1}$, Nagehan Emiralioğlu², Güzin Cinel², Ebru Yalçın², Deniz Doğru², \\ Nural Kiper², Hayriye Uğur Özçelik ${ }^{2}$ \\ ${ }^{2}$ Division of Pediatric Pulmonology, ${ }^{1}$ Department of Pediatrics, Hacettepe University Faculty of Medicine, Ankara, Turkey. \\ E-mail: etekbas.tekbas@gmail.com
}

Received: 31st December 2017, Accepted: 19th April 2018

SUMMARY: Güney E, Emiralioğlu N, Cinel G, Yalçın E, Doğru D, Kiper N, Özçelik HU. Nasal nitric oxide levels in primary ciliary dyskinesia, cystic fibrosis and healthy children. Turk J Pediatr 2019; 61: 20-25.

Primary ciliary dyskinesia (PCD) is a rare, inherited disorder characterized by recurrent respiratory tract infections. The measurement of nasal nitric oxide (nNO) is an important test for the diagnosis of PCD. In this study, we aim to evaluate NIOX-MINO ${ }^{\circledR}$, which is an easily applicable method for measuring nNO, in the diagnosis of patients with PCD and define diagnostic cut-off levels. Furthermore, determining the normal limits of nNO in healthy children and investigating nNO levels of children with cystic fibrosis (CF) are the other aims of this study. The children included in this study were 5 to 18.5 years old, 46 of them had PCD, 44 had CF and 200 were healthy children. To our knowledge, this work contains the widest population compared to previous studies. Subjects receiving steroids or antibiotics or those with any acute respiratory tract infection, asthma or allergic rhinitis were not included in the study. Mean nNO levels were found as 10.4, 22.8 and 21.0 $\mathrm{ppb}$ in PCD, CF and healthy children, respectively. The nNO levels for PCD patients were found significantly lower than children with CF and the control groups $(\mathrm{p}<0.05)$. In this study, the diagnostic nNO cut-off level between PCD and the other two groups was determined to be $<11.5 \mathrm{ppb}$ with $\% 83.6$ specificity and \%67.4 sensitivity. The screening of nNO with NIOX-MINO method provides early diagnose before mucosal biopsy of patients who are suspected to have PCD and therefore, prevents co-morbidities and prolongs survival with early treatment.

Key words: primary ciliary dyskinesia, cystic fibrosis, nitric oxide, early diagnosis, case control studies.

Primary ciliary dyskinesia (PCD) is a rare $(1$ in 20.000-60.000 live births), typically autosomal recessive inherited disease associated with defective ciliary structure and/or function that results in abnormal mucociliary clearance. ${ }^{1,2}$ In the 1930s, triad of situs inversus totalis, bronchiectasis and sinusitis was defined as Kartagener's Syndrome. ${ }^{3}$ Afzelius ${ }^{4}$ showed that ciliary abnormalities of the bronchial cilia and sperm flagella bring about chronic sinopulmonary infections and male infertility. Later, all cilia ultrastructural abnormalities similar to the ones defined above were literally termed as immotile cilia syndrome. Impaired function of cilia affects the heart and abdominal organs laterality during embryonic development. Situs inversus totalis constitutes approximately half of the cases of PCD. ${ }^{5}$ The symptoms of PCD such as rhinitis, secretory otitis media, cough and recurrent bronchitis are common to many other diseases which often yield late diagnosis of PCD. ${ }^{6}$ The major complication of PCD is bronchiectasis which can be prevented by early diagnosis and treatment.

Nitric oxide (NO) is a cellular signaling 
molecule, functioned as modulator of ciliary function, vasodilatation, bronchodilatation, platelet aggregation, neurotransmission and immune function. ${ }^{7,8}$ Firstly, Gustafsson et al. ${ }^{9}$ showed NO presence in exhaled breath of humans in 1991. Nasal nitric oxide (nNO) is synthesized in respiratory airways, particularly in the paranasal sinuses. ${ }^{10}$ The nNO level is low in individuals with PCD; thereby it can be helpful in the diagnosis before biopsy. Previously, chemiluminiscence NO analyzer was used for assessing nNO levels, however, this instrument is expensive, large and immobile. Recent studies about NIOX-MINO ${ }^{\circledR}$ (Aerocrine $A B$, Sweden), which is a hand-held, noninvasive device, showed that it is useful, reliable, rapid and has a correlation with chemiluminescence NO analysis. ${ }^{11,12}$

Our objectives were measuring and comparing nNO levels between children with PCD, cystic fibrosis (CF) and healthy individuals via NIOX$\mathrm{MINO}^{\circledR} \mathrm{NO}$ analyzer, assessing healthy children normal levels, and cut-off nNO levels for PCD.

\section{Material and Methods}

\section{Study Population}

In this study, children diagnosed with PCD (including Kartagener's syndrome) and CF were recruited from Hacettepe University İhsan Doğramacı Children Hospital Respiratory Clinic. During the study period, PCD patients who accepted participation in the study were diagnosed by compatible history and symptoms, radiological findings, ciliary ultrastructure abnormalities by using electron microscopy and/or analysis of ciliary beat pattern and frequency abnormalities by using high speed video microscopy. CF was diagnosed by suggestive history, increased sweat test and/or genotyping. In this study out of 290 individuals, 46 children had PCD, 44 had CF and 200 were healthy subjects. The age of participants were distributed through 5-18.5 years. All participants were examined for nasal anatomic defects and polyps. If any of them existed in one part of the nose, the other part of the nose was preferred. Furthermore, the subjects included in this study were not receiving steroids or antibiotic and had no signs of an acute respiratory tract infection, asthma or allergic rhinitis.
Nasal NO measurements In our study, nNO was measured during quiet exhalation with NIOX$\mathrm{MINO}^{\circledR}$. During the tests, flow rate was $5 \mathrm{~mL} / \mathrm{s}$ and a nasal olive was put into one nostril and the other nostril was closed. After the nasal olive was removed from the nostril, the time bar was filled approximately in $45 \mathrm{~s}$ for $5 \mathrm{~mL} / \mathrm{s}$ flow rate and thus nNO measurements resulted in a few minutes. Since $5 \mathrm{~mL} / \mathrm{s}$ flow rate yields faster results over $2 \mathrm{~mL} / \mathrm{s}$, we preferred the former one. This method was easily performed by children; therefore, it was more practical. nNO was measured by well-trained department technicians while participants were in sitting position. NO level interval was $5-1700 \mathrm{ppb}$ by NIOX-MINO ${ }^{\circledR}$ device and NO level $<5 \mathrm{ppb}$ couldn't be measured as a unique number. In such circumstances, NO level $<5$ ppb were accepted as $2.5 \mathrm{ppb}$ according to the mean value between 0 and 5 with the suggestion from the statistics department.

In order to reduce the effect of air pollution, nNO was measured in May-September 2012, in which the heating systems were not used. Many environmental factors affect nNO levels, therefore, for obtaining standardization, mobile phones were closed or moved away from the NIOX-MINO ${ }^{\circledR}$ device and an isolated and ventilated room was preferred when making nNO measurement.

\section{Statistics}

The data was evaluated by IBM SPSS version 21 . Data was presented with descriptive statistics such as mean, median, standard deviation, minimum, maximum numbers and percentages. Kruskal-Wallis and Mann-Whitney U tests were used for numerical values. Sensitivity, specificity and receiver operating characteristic (ROC) analysis were utilized to find best cut-off value between groups. $\mathrm{P}<0.05$ was statistical significance level.

The study was approved by Hacettepe University Research Ethics Committee on 11.05.2012 with report number HEK 12/71. Before the measurement of nasal $\mathrm{NO}$, all subjects and parents gave written informed consent to participate in this study.

\section{Results}


Table I. Nasal NO Levels of PCD, CF and Control Groups.

\begin{tabular}{lcccccc}
\hline Groups & $\mathrm{n}$ & Mean $(\mathrm{ppb})$ & $\mathrm{SD}(\mathrm{ppb})$ & $\begin{array}{c}\text { Median } \\
(\mathrm{ppb})\end{array}$ & $\begin{array}{c}\text { Minimum } \\
(\mathrm{ppb})\end{array}$ & $\begin{array}{c}\text { Maximum } \\
(\mathrm{ppb})\end{array}$ \\
\hline PCD & 46 & 10.4 & 8.3 & 9.0 & 2.5 & 42.0 \\
CF & 44 & 22.8 & 18.7 & 17.0 & 2.5 & 81.0 \\
Control & 200 & 21.0 & 8.9 & 20.0 & 7.0 & 48.0
\end{tabular}

NO: nitric oxide, PCD: primary ciliary diskinesia, CF: cystic fibrosis, ppb: parts per billion

\section{Participants}

In total, 290 subjects were included in this study. One hundred and thirty-four (46.2\%) of them were girls and 156 of them (53.8\%) were boys. In the PCD group, we had 46 individuals, $23(50 \%)$ participants were girls and $23(50 \%)$ were boys. In the CF group we had 44 individuals, $22(50 \%)$ participants were girls and $22(50 \%)$ were boys. In the control group we had 200 individuals, 89 (44.5\%) of them were girls and $111(55.5 \%)$ of them were boys. There was no statistically significant difference between PCD, CF and control group concerning gender $(p=0.685)$.

Population's age range was 5-18.5 years and the mean values for PCD, CF, healthy controls were $11.9,10.2$ and 11.1 years, respectively. There was no statistically significant difference between groups concerning ages $(p=0.053)$. Mean diagnostic ages of PCD patients were 7.6 years and none of them were diagnosed in the newborn period. Mean diagnostic ages of CF patients were 1.8 years, furthermore 4 of them were diagnosed in the newborn period and 1 of them had a prenatal diagnose because of family's CF history.

Pulmonary function tests were successfully performed by 38 PCD and 33 CF patients. The mean and standard deviation (SD) of PCD patients for Forced expiratory volume in 1 second (FEV1), Forced vital capacity (FVC), FEV1/FVC, expiratory flow between $25 \%$ and
$75 \%$ of vital capacity (FEF25-75) were $78.4 \pm$ $19.1,84.8 \pm 17.5,87.4 \pm 11.1,58.3 \pm 21.7$, respectively. The mean and $\mathrm{SD}$ of $\mathrm{CF}$ patients for FEV1, FVC, FEV1/FVC, FEF25-75 were $83.7 \pm 15.9,90.9 \pm 15.8,92.0 \pm 8.5,73.0$ \pm 25.7 , respectively. There was no statistically significant difference between PCD and CF groups concerning pulmonary function test results except FEF25-75 parameter.

Nasal NO levels Mean, SD, median, minimum and maximum values of $\mathrm{nNO}$ are shown in Table I for all groups. Mean nNO levels for children with PCD, CF and healthy controls were respectively $10.4 \pm 8.3,22.8 \pm 18.7$ and $21 \pm 8.9 \mathrm{ppb}$. nNO was statistically significantly lower in PCD when compared to $\mathrm{CF}$ and healthy controls $(p<0.05)$ and there was no statistically significant difference between CF and the control group.

In our study, the nNO cut-off level was $<11.5$ ppb (sensitivity: \%67.4, specificity: \%83.6) when we compared PCD versus both control and $\mathrm{CF}$ groups. The nNO cut-off level was found $<11.5 \mathrm{ppb}$ (sensitivity: \%67.4, specificity: \%87.5) between PCD and control group. The nNO cut-off level was $<10.5 \mathrm{ppb}$ (sensitivity: \%72.7, specificity: \%63.0) between PCD and the CF groups. The cut-off, sensitivity and specificity values are also summarized in Table II.

ROC curves denote sensitivity and 1- specificity values for nNO levels. The more the area

Table II. Nasal NO Cut-off Values of PCD, CF and Control Groups.

\begin{tabular}{lccc}
\hline & Cut-off $(\mathrm{ppb})$ & Specificity $(\%)$ & Sensitivity (\%) \\
\hline PCD vs CF and Control & 11.5 & 83.6 & 67.4 \\
PCD vs Control & 11.5 & 87.5 & 67.4 \\
PCD vs CF & 10.5 & 63.0 & 72.7 \\
\hline
\end{tabular}

NO: nitric oxide, PCD: primary ciliary diskinesia, CF: cystic fibrosis, ppb: parts per billion 


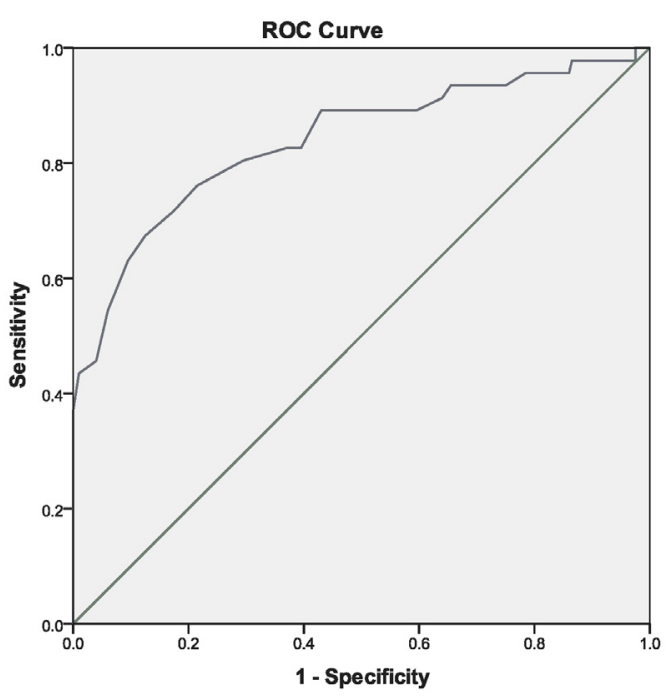

Fig. 1. ROC Curve for nNO Levels of PCD and Control Groups: Area under the ROC curve: 0.84, p: 0.00, asymptotic $95 \%$ confidence interval; lower bound: 0.76 , upper bound: 0.91

under the ROC curve, the more significant the result is. The ROC curves obtained from the comparison of nNO levels of PCD versus healthy and $\mathrm{CF}$ are shown in Figure 1 and Figure 2, respectively. Moreover, Figure 3 presents the ROC curve obtained from the comparison of PCD versus both the healthy and CF. Note that, the results are found statistically significant since all of the ROC curves have $\mathrm{p}<0.05$.

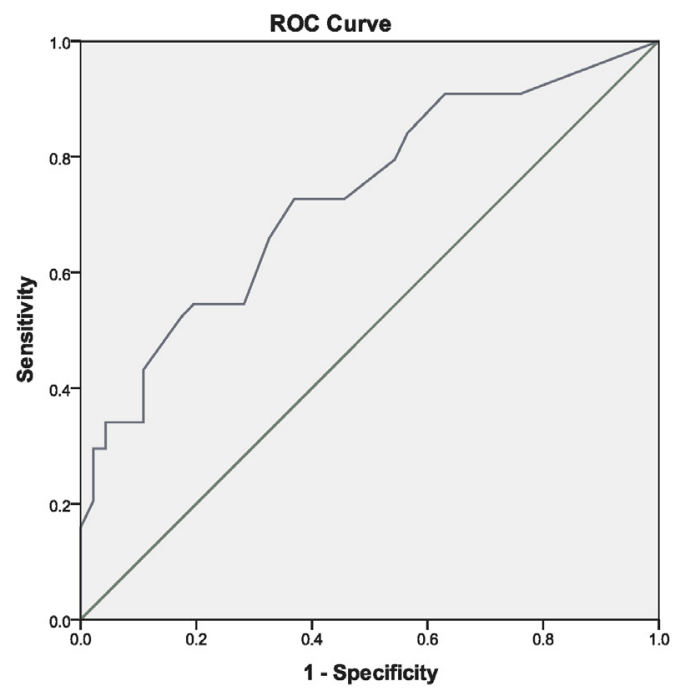

Fig. 2. ROC Curve for nNO Levels of PCD and CF Groups: Area under the ROC curve: 0.73 , p: 0.00 , asymptotic $95 \%$ confidence interval; lower bound: 0.62 , upper bound: 0.83

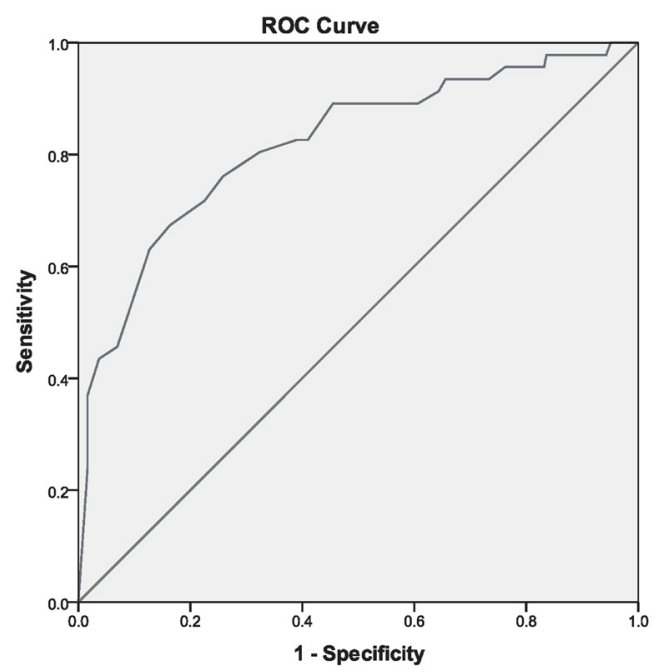

Fig. 3. ROC Curve for nNO Levels of PCD versus both the Control and CF Groups: Area under the ROC curve: 0.82 , p: 0.00 , asymptotic $95 \%$ confidence interval; lower bound: 0.74 , upper bound: 0.89

\section{Discussion}

Diagnosis of PCD is often delayed because of symptoms being common to many other diseases. Clinical symptoms, family history, sputum culture results, radiographic findings, nNO levels, analysis of ciliary ultrastructure with electron microscopy, analysis of ciliary beat pattern and frequency with high speed video microscopy and genetic tests are utilized to diagnose PCD. Some studies have shown that $\mathrm{nNO}$ levels are very low in PCD. ${ }^{13,14}$

Since 2009, nNO measurement have been used for screening PCD patients in Europe. ${ }^{15,16}$ In the literature, there exist many studies that measured $\mathrm{nNO}$ with chemiluminescence method and NIOX-MINO ${ }^{\circledR}$ for PCD, CF and healthy subjects and moreover they have determined correlation between these methods. ${ }^{17-21}$ Montella et al. ${ }^{17}$ used nasal quiet exhalation method and $0,05 \mathrm{~L} / \mathrm{s}$ flow rate with $\mathrm{NIOX}-\mathrm{MINO}^{\circledR}$ for measurement of $\mathrm{nNO}$ for $14 \mathrm{PCD}, 11 \mathrm{CF}$ and 13 healthy control group subjects. nNO cut-off value was determined as $<23 \mathrm{ppb}$ with $100 \%$ sensitivity, \%85 specificity between PCD and control groups, $<26 \mathrm{ppb}$ with $100 \%$ sensitivity, $91 \%$ specificity between PCD and CF groups and $<57$ ppb with $82 \%$ sensitivity, $31 \%$ specificity between $\mathrm{CF}$ and control groups. In the same study, when $0.05 \mathrm{~L} / \mathrm{s}$ flow rate and humming nasal exhalation method was used, PCD and 
control groups were compared and nNO cutoff was demonstrated as <50 ppb with $100 \%$ sensitivity, $100 \%$ specificity. In addition, PCD and $\mathrm{CF}$ groups were compared and cut-off value was $<21 \mathrm{ppb}$ with $93 \%$ sensitivity, $91 \%$ specificity. This study showed that nNO values were influenced by the measurement technique, thus nNO cut-off values may vary for each method.

In our study, nasal quiet exhalation method was chosen because of its easy use, and also nNO values were found to be lower than previous studies. nNO cut-off values were determined as $<11.5 \mathrm{ppb}$ with $67.4 \%$ sensitivity, $83.6 \%$ specificity when we compared PCD versus control and CF groups, $<11.5$ ppb with $67.4 \%$ sensitivity, $87.5 \%$ specificity between PCD and control groups and $<10.5 \mathrm{ppb}$ with $72.7 \%$ sensitivity, $63.0 \%$ specificity between PCD and $\mathrm{CF}$ groups. In our study, we found our cut-off nNO levels using same measurement technique for a lot of healthy subjects, PCD and CF patients. As far as we know, this work has the largest study group of healthy and PCD children for measuring nNO levels with NIOX MINO ${ }^{\circledR}$ method. In our study, nNO levels were lower in PCD compared to other groups which is consistent with the previous studies $17,21,22$. However, our sensitivity and specificity values were lower than the other studies. Previous studies found that nasal quiet exhalation method with chemiluminescence analyzer had the lowest nNO values compared to the other nNO measurement methods. ${ }^{23}$ Lower respiratory tract contamination is much more effective for this method than other methods. Note that, nNO levels in lower respiratory tract are lower than upper respiratory tract and paranasal sinuses. Therefore, the large study population and the method we used might have a role for the low values of $\mathrm{nNO}$ measurements.

We had some limitations such as some of the PCD and CF patients had positive sputum culture and/or colonization. In spite of that, none of the participants in the whole population had any signs of an acute infection in the 4 weeks period before the nNO measurements. In addition, NIOX-MINO ${ }^{\circledR}$ device cannot measure nNO levels $<5 \mathrm{ppb}$. Therefore, we accepted $2.5 \mathrm{ppb}$ for each nNO level $<5 \mathrm{ppb}$ according to the recommendation of the department of statistics at our university.

The common symptoms frequently cause the diagnosis of PCD to be delayed. Effective and intensive care enable the respiratory function to be stabilized and hence preventing complications for many years. History, physical examination, radiological and microscopic findings guide diagnosing PCD. Despite the fact that nNO levels obtained in this study do not show high sensitivity, this study supports the diagnosis of PCD by measuring nNO levels before the use of mucosal brush or biopsy.

\section{Acknowledgement}

We wish to thank Sultan Ünal Dinçel, Meltem Şahin and Nermin Gürcan for their invaluable contributions and Teknikel Corporation for kit donation during this study.

Funding source: Hacettepe University Scientific Research and Development Office Project Number: 010A101008 and Teknikel Corporation by means of kit donations.

\section{REFERENCES}

1. O'Callaghan C, Chilvers M, Hogg C, Bush A, Lucas J. Diagnosing primary ciliary dyskinesia. Thorax 2007; 62: 656-657.

2. Zariwala MA, Omran H, Ferkol TW. The emerging genetics of primary ciliary dyskinesia. Proc Am Thorac Soc 2011; 8: 430-433.

3. Kartagener M. Zur pathogenese der bronkiectasien: bronkiectasien bei situs viscerum inversus. Beitr Klin Tuberk 1933; 82: 489-501.

4. Afzelius BA. A human syndrome caused by immotile cilia. Science 1976; 193: 317-319.

5. Eliasson R, Mossberg B, Camner P, Afzelius BA. The immotile-cilia syndrome: a congenital ciliary abnormality as an etiologic factor in chronic airway infections and male sterility. N Engl J Med 1977; 297: $1-6$.

6. Bush A, Chodhari R, Collins $\mathrm{N}$ et al. Primary ciliary dyskinesia: current state of the art. Arch Dis Child 2007; 92: 1136-1140.

7. Ignarro LJ, Buga GM, Wood KS, Byrns RE, Chaudhuri G. Endothelium-derived relaxing factor produced and released from artery and vein is nitric oxide. Proc Natl Acad Sci 1987; 84: 9265-9269.

8. Ricciardolo FL, Di Stefano A, Sabatini F, Folkerts G. Reactive nitrogen species in the respiratory tract. Eur J Pharmacol 2006; 533: 240-252.

9. Gustafsson LE, Leone A, Persson M, Wiklund N, Moncada S. Endogenous nitric oxide is present in the exhaled air of rabbits, guinea pigs and humans. Biochem Biophys Res Commun 1991; 181: 852-857. 
10. Lundberg JON, Rinder J, Weitzberg E, Lundberg JM, Alving K. Nasally exhaled nitric-oxide in humans originates mainly in the paranasal sinuses. Acta Physiol Scand 1994; 152: 431-432.

11. Alving K, Janson C, Nordvall L. Performance of a new hand-held device for exhaled nitric oxide measurement in adults and children. Respir Res 2006; 7: 67.

12. Schiller B, Hammer J, Barben J, Trachsel D. Comparability of a hand-held nitric oxide analyser with online and offline chemiluminescence-based nitric oxide measurement. Pediatr Allergy Immunol 2009; 20: 679-685.

13. Noone PG, Leigh MW, Sannuti A et al. Primary ciliary dyskinesia: diagnostic and phenotypic features. Am J Respir Crit Care Med 2004; 169: 459-467.

14. Karadag B, James A, Gültekin E, Wilson N, Bush A. Nasal and lower airway level of nitric oxide in children with primary ciliary dyskinesia. Eur Respir J 1999; 13: 1402-1405.

15. Barbato A, Frischer T, Kuehni CE et al. Primary ciliary dyskinesia: a consensus statement on diagnostic and treatment approaches in children. Eur Respir J 2009; 34: 1264-1276.

16. Hogg C. Primary ciliary dyskinesia: when to suspect the diagnosis and how to confirm it. Paediatr Respir Rev 2009; 10: 44-50.
17. Montella S, Alving K, Maniscalco M et al. Measurement of nasal nitric oxide by hand-held and stationary devices. Eur J Clin Invest 2011; 41: 1063-1070.

18. Maniscalco M, de Laurentiis G, Weitzberg E, Lundberg JO, Sofia M. Validation study of nasal nitric oxide measurements using a hand-held electrochemical analyser. Eur J Clin Invest 2008; 38: 197-200.

19. Corbelli R, Bringolf-Isler B, Amacher A, Sasse B, Spycher M, Hammer J. Nasal nitric oxide measurements to screen children for primary ciliary dyskinesia. Chest 2004; 126: 1054-1059.

20. Piacentini GL, Bodini A, Peroni D et al. Nasal nitric oxide for early diagnosis of primary ciliary dyskinesia: practical issues in children. Respir Med 2008; 102: 541-547.

21. Walker WT, Liew A, Harris A, Cole J, Lucas JS. Upper and lower airway nitric oxide levels in primary ciliary dyskinesia, cystic fibrosis and asthma. Respir Med 2013; 107: 380-386.

22. Wodehouse T, Kharitonov S, Mackay I, Barnes P, Wilson $\mathrm{R}$, Cole P. Nasal nitric oxide measurements for the screening of primary ciliary dyskinesia. Eur Respir J 2003; 21: 43-47.

23. de Winter-de Groot, van der Ent CK. Measurement of nasal nitric oxide: evaluation of six different sampling methods. Eur J Clin Invest 2009; 39: 72-77. 